Social mobility theory suggests greater economic mobility will moderate the association of childhood adversity with adult functional limitations. We examined this theory using the Panel Study of Income Dynamics (1992-2015) and the 2014 PSID Childhood Retrospective Circumstances Study ( $\mathrm{n}=4,081 ; 15,648$ status transitions). The outcome indicated impairment in one or more activities of daily living (ADLs), measured by the percentage of years lived from age 40 with ADL impairment. Four or more of nine adversities defined high adversity. For each observation beginning at age 40 we averaged the preceding 20 years of income, using ratios of annual household income to the annual poverty threshold. The ratio of the average of the last 5 years' income to the first 5-year average measured economic mobility. Multinomial logistic Markov models estimated annual disability transition probabilities, adjusting age, sex, race, education, income level, and economic mobility. Using the probabilities we created large populations with microsimulation, measuring annual ADL disability for each individual through death. Women exemplify results: ADL disability varied greatly, from $5.5 \%$ of remaining life with postsecondary education, the highest income decile, and highest mobility, to $43.2 \%$ with less than high school and the lowest income and mobility. Women reporting high adversity had more adult ADL impairment than others $(\mathrm{p}<0.001)$; economic mobility significantly moderated that association among women without postsecondary education and below median income; women in the lowest income decile were impaired $42.6 \%$ with low mobility, $35.7 \%$ with high mobility $(\mathrm{p}<0.001)$. Economic mobility significantly moderates the association of childhood adversity with later-life health.

\section{HIGH FLOURISHING IS LINKED WITH LOW LIFE COURSE DISABILITY: RESULTS FROM TWO DECADES OF PANEL DATA}

\section{S. Laditka ${ }^{1}$, J. Laditka ${ }^{2}, 1$. University of North Carolina at} Charlotte, 2. University of North Carolina

Flourishing, a measure of well-being characterized by supportive relationships, contributing to the happiness of others, having purpose and meaning in life, and being optimistic, is associated with better health. Flourishing may promote healthy behaviors, help manage chronic diseases, and control stress. Flourishing-related traits typically remain stable after about age 25. With data from the Panel Study of Income Dynamics (PSID) and the 2016 PSID Well Being and Daily Life Supplement (1992-2013, n=7,197; 33,024 functional status transitions), we examined associations of a 7-item flourishing scale with three health outcomes: difficulty with activities of daily living (ADLs, not having help), ADL dependency (usually having help), and impairment in instrumental ADLs (IADLs). Multinomial logistic Markov models estimated annual probabilities of transitioning among IADL and ADL status states, adjusted for age and education, with estimates for African American, Hispanic, and white women and men. Using the probabilities, we created large populations with dynamic microsimulations, each individual having a known functional status each year, age 25 to death. In all groups people with low flourishing had significantly more IADL impairment, ADL disability, and ADL dependency throughout adult life than those with high flourishing $(\mathrm{p}<0.01)$. Illustrating the results, among Hispanic women at age 50 with low flourishing, $8.8 \%, 9.8 \%$, and $6.3 \%$ had
IADL impairments, ADL disability, and ADL dependency, respectively, compared with $5.0 \%, 4.2 \%$, and $2.3 \%$ with high flourishing (all $\mathrm{p}<0.01)$. It may be useful to develop interventions to promote behaviors, beliefs, and attitudes that are associated with flourishing to maintain or improve functional status.

\section{IS HIGHER LEVEL OF SOCIAL SUPPORT ASSOCIATED WITH LOWER ODDS OF ELDER ABUSE AMONG U.S. CHINESE OLDER ADULTS?}

S. Zheng ${ }^{1}$, X. Dong ${ }^{2}$, 1. Rush Institute of Healthy Aging, Rush University Medical Center, 2. Rush University Medical Center

Research has examined the relationship between the positive social support (PSS) and elder abuse (EA) but less have examined the negative aspect of social support (NSS), especially among minority groups. This study aims to investigate the relationships between two aspects of social support and EA among U.S. Chinese older adults. The data were drawn from the PINE study with a representative sample of Chinese older adults aged 60 years or older in Greater Chicago area ( $\mathrm{N}=3157)$. We used modified VASS instrument (10 items) to assess EA. PSS and NSS from spouse/family members/ friends were measured by a 12 -item scale. Multivariate logistic regression analyses were conducted to test the relationships. After adjusting for confounders, the higher level of PSS from all sources (OR, $0.88[0.85,0.91])$, from spouse $(\mathrm{OR}$, $0.70[0.64,0.76])$, and from family members (OR, $0.73[0.68$, $0.79]$ ) were associated with lower likelihood of EA, but PSS from friends had a non-significant relationship. Participants with a higher level of NSS from all sources (OR, 1.51[1.41, 1.61]), from spouse (OR, 1.84[1.64, 2.07]), from family members (OR, 2.36[2.03, 2.75]), and from friends (OR, $1.69[1.32,2.17])$ were more likely to experience EA. The results showed that higher level of social support does not always predict lower odds of EA. Future qualitative or longitudinal research needs to explore detailed cultural explanations and casual relationships.

\section{LONGITUDINAL IMPLICATIONS OF SOCIAL INTEGRATION FOR AGE AND GENDER DIFFERENCES IN LATE-LIFE PHYSICAL FUNCTIONING}

M. Toyama, J. Owino, H. Fuller, North Dakota State University

Aging adults may face limitations in physical functioning which can influence their quality of life. Though previous research has documented potential benefits of social integration (SI) for late-life physical functioning, it is not well understood how those benefits may vary over time among women and men of different ages. The present study examined potential age and gender differences in the longitudinal associations between SI and physical functioning by constructing a two-level hierarchical linear model with three waves of data collected over four years from a sample of 403 adults (baseline age of 60 to 98). Whereas age and SI predicted functional limitations, findings also indicated multiple interactions of age, gender, and SI on levels and trajectories of functional limitations. Older people with low SI appeared to experience greater increase in functional limitations than those with high SI, a pattern not found among 
younger people. Levels and trajectories of functional limitations also differed between genders. Older women showed greater increases in functional limitations than older men in contrast to relatively similar trajectories between younger women and men. In addition, having high levels of SI was associated with fewer functional limitations among men as compared to women. These findings suggest that SI may be more protective for late-life physical functioning among men as well as older cohorts of both genders. This study highlights future directions for longitudinal research on SI in late-life. Moreover, this study highlights the need to explore practical implications for focusing on promoting SI among the oldest old.

\section{OBJECTIVE PHYSICAL FUNCTION AND MENTAL HEALTH-RELATED OUTCOMES IN OLDER PRISONERS \\ L. Barry ${ }^{1}$, D. Wakefield ${ }^{2}$, Y. Conwell ${ }^{3}$, D. Steffens ${ }^{4}, 1$. University of Connecticut Center on Aging, 2. UCONN Center on Aging, 3. University of Rochester School of Medicine, Rochester, NY, 4. Department of Psychiatry, University of Connecticut Health Center}

The U.S. prison population is aging; increasing numbers of older adults (age $\geq 50$ ) are being incarcerated and are aging “in place." Older prisoners are likely to experience depression and suicidal ideation (SI), which are both strong predictors of suicide. However, "conventional" factors associated with increased suicide risk in mixed-age inmate samples (e.g., time served or single-cell housing) appear less relevant for older inmates. We sought to determine if physical function was associated with depression and SI in older prisoners. As part of an ongoing study, we conducted face-to-face interviews with 132 male prisoners age $\geq 50$ from 4 Connecticut state prisons. Physical function was assessed objectively using the Short Physical Performance Battery (SPPB). The outcomes, depression and SI, were assessed using the PHQ-9 (range 0-27) and the Geriatric Suicide Ideation Scale (range 31-105); higher scores on each scale indicated worsening severity. Participants were racially diverse (42\% White, 39\% Black, 20\% Hispanic/ Other) and had a mean age of $57.2 \pm 6.6$ years (range 50-79 years). Using linear regression models controlling for age group (50's vs 60+), race, years in prison, and ADL difficulties, we found that increasing SPPB total score was negatively associated with depression (beta $=-1.18 \pm 0.30 ; \mathrm{p}=0.0001)$, SI (beta $=-0.74 \pm 0.34 ; \mathrm{p}=0.03$ ), and GSIS total score (beta $=$ $-2.62 \pm 0.97 ; \mathrm{p}=0.008)$. Physical function is strongly associated with mental health outcomes in older prisoners. Using objective indicators to assess older prisoners' physical function may help to identify those who may be at higher risk of depression and SI and may indicate a target area for intervention.

\section{SESSION 1750 (POSTER)}

\section{DEPRESSION AND PSYCHOSOCIAL CHARACTERISTICS II}

\section{AGING ANXIETY MEDIATES THE RELATIONSHIPS OF KNOWLEDGE OF AGING AND CONTACT WITH OLDER ADULTS TO AGEIST ATTITUDES}

G. Caskie, H. Bashian, A. Canell, Lehigh University
Prior research has indicated that lack of knowledge about aging and little contact with older adults are related to more ageist attitudes about older adults. Based on the principles of Terror Management Theory (e.g., Martens, Goldenberg, \& Greenberg, 2005), we hypothesized that anxiety about aging may mediate, or explain, these relationships. A sample of 483 participants (18-55 years; 244 males, 239 females) completed survey measures online including a demographic form, the Facts on Aging Quiz, Contact with Older Adults Scale, Aging Anxiety Scale, and Fraboni Scale of Ageism. Including age as a covariate, the multiple regression model explained $20.8 \%$ of the variance in ageist attitudes $(\mathrm{p}<.0001)$; three of the four predictors of more ageist attitudes about older adults were statistically significant: less knowledge of aging ( $b=-.64, p<.0001)$, greater anxiety $(b=.35, p<.0001)$, and younger age $(b=-.14$, $\mathrm{p}=.0004)$. Amount of contact was not a significant predictor $(b=.004, p=.912)$. However, $95 \%$ bootstrap confidence intervals indicated that both of the hypothesized indirect effects were statistically significant: (1) knowledge to anxiety to ageist attitudes (point estimate $=-.135$, 95\% CI of -.220 to -.065$)$ and (2) contact to anxiety to ageist attitudes (point estimate $=-.046,95 \%$ CI of -.077 to -.019 ). Thus, aging anxiety mediates the relationships of knowledge about aging and contact with older adults to ageist attitudes. One implication of these results is that interventions to reduce ageist attitudes, particularly if they involve increasing contact with older adults, may be most successful if they also work to reduce individuals' anxiety about their own aging.

\section{ALL BEHAVIOUR HAS MEANING: CONCEPTUALIZING CHALLENGING ASSUMPTIONS IN DEMENTIA CARE}

K. Aubrecht, J. Keefe, Mount Saint Vincent University

"All behaviour has meaning" is one of the central tenants of dementia care research and education. Yet, the question, "What is behaviour?" is often left unasked within dementia care research, education, practice and policy. Drawing from perspectives in cultural gerontology, interpretive sociology and critical disability studies, this presentation theorizes 'challenging' assumptions about behaviour in discourses of dementia care. Our constructivist conceptual analysis of behaviour leads to a consideration of the behaviourist tradition in psychology, and the biopsychosocial model of dementia care and Thomas Kitwood's and Carl Rogers' seminal works on meaning, personhood and relationships. Within this tradition and these works, what behaviour means and why it matters is informed by ideas about adaptation and adjustment; concepts which posit behaviour as an expression (and measure) of the 'person-environment fit', and by virtue of that, a means of recognizing some people as out of place. Through unpacking the meaning of behaviour as a 'challenging assumption' in dementia care, we trace the relations between behaviour and personality. We contend that scholarly work of this nature within gerontology is critical to understanding the social significance of personcentred dementia care, and the challenges and opportunities a behaviourist approach poses for recognizing personhood in dementia. 